Acta Crystallographica Section B

Structural

Science

ISSN 0108-7681

\section{Toshiyuki Matsunaga, ${ }^{a *}$ Rie Kojima, ${ }^{\text {b Noboru Yamada, }}{ }^{c}$ Yoshiki Kubota $^{d}$ and Kouichi Kifune ${ }^{\mathrm{e}}$}

a Device Solutions Center, R\&D Division, Panasonic Corporation, 3-1-1 Yagumo-Nakamachi, Moriguchi, Osaka 570-8501, Japan, bAVC Networks Company, Panasonic Corporation, 115 Matsuo-cho, Kadoma, Osaka 571-8504, Japan, 'Department of Materials Science \& Engineering, Kyoto University, Yoshidahonmachi, Sakyo-ku, Kyoto 606-8501, Japan, ${ }^{\mathbf{d} G r a d u a t e}$ School of Science, Osaka Prefecture University, 1-1 Gakuen-cho, Sakai, Osaka 5998531, Japan, and ${ }^{\mathbf{e}}$ Faculty of Liberal Arts and Sciences, Osaka Prefecture University, 1-1 Gakuen-cho, Sakai, Osaka 599-8531, Japan

Correspondence e-mail: matsunaga.toshiyuki@jp.panasonic.com

\title{
Structural transformation of Sb-based high-speed phase-change material
}

The crystal structure of a phase-change recording material (the compound $\mathrm{Ag}_{3.4} \mathrm{In}_{3.7} \mathrm{Sb}_{76.4} \mathrm{Te}_{16.5}$ ) enclosed in a vacuum capillary tube was investigated at various temperatures in a heating process using a large Debye-Scherrer camera installed in BL02B2 at SPring-8. The amorphous phase of this material turns into a crystalline phase at around $416 \mathrm{~K}$; this crystalline phase has an A7-type structure with atoms of Ag, In, Sb or Te randomly occupying the $6 c$ site in the space group. This structure was maintained up to around $545 \mathrm{~K}$ as a single phase, although thermal expansion of the crystal lattice was observed. However, above this temperature, phase separation into $\mathrm{AgInTe}_{2}$ and $\mathrm{Sb}-\mathrm{Te}$ transpired. The first fragment, AgInTe 2 , reliably maintained its crystal structure up to the melting temperature. On the other hand, the atomic configuration of the $\mathrm{Sb}-\mathrm{Te}$ gradually varied with increasing temperature. This gradual structural transformation can be described as a continuous growth of the modulation period $\gamma$.

\section{Introduction}

Phase change recording is now extensively used for highdensity non-volatile memories (Wuttig \& Yamada, 2007). Since the 1970s, various materials have been proposed for this purpose, and today we have obtained two superior materials: GeTe- $\mathrm{Sb}_{2} \mathrm{Te}_{3}$ (GST) (Yamada et al., 1991) and Sb-Te-based alloys such as $\mathrm{Ag}_{3.4} \mathrm{In}_{3.7} \mathrm{Sb}_{76.4} \mathrm{Te}_{16.5}$ (AIST or silver indium antimony tellurium; Iwasaki et al., 1992); these materials are now practically used as the memory layers in phase-change optical disk media, as well as in the memory cells of solid-state electrical memories. Recording can be achieved using laser irradiation or ohmic heating to cause reversible phase changes between the amorphous and crystalline phases. We analyzed the crystal structure of AIST about 10 years ago (Matsunaga et al., 2001), revealing that AIST has an A7-type structure, the same as that of pure $\mathrm{Sb}$, in which four elements randomly occupy the $6 c$ site in the space group. It has been presumed that this simple and spatially isotropic $p-p$ connected sixcoordination structure enables instantaneous transformation from the amorphous phase to the crystalline phase by minimal atomic rearrangement (Matsunaga et al., 2006; Matsunaga, Yamada et al., 2011). Our analysis also revealed that this A7type quaternary crystal held its rhombohedral structure, showing a continuous atomic shift along the axis from $z=$ 0.233 to $1 / 4$, when the temperature was raised close to the melting point. We examined the crystal structures of $\mathrm{Sb}-\mathrm{Te}$ binary compounds, which are the mother alloys of the AIST materials, as well as those of $\mathrm{Bi}-\mathrm{Te}$ compounds. These compounds are known, in thermal equilibrium, to have a series of commensurately or incommensurately modulated long-period layer structures, depending on their binary
Received 28 March 2012

Accepted 20 September 2012

B-IncStrDB Reference: 6732EXfQbW 
compositions, between $\mathrm{Sb}$ and $\mathrm{Sb}_{2} \mathrm{Te}_{3}$ or between $\mathrm{Bi}$ and $\mathrm{Bi}_{2} \mathrm{Te}_{3}$. Our present investigation of an as-deposited $\mathrm{Ag}_{3.4} \mathrm{In}_{3.7} \mathrm{Sb}_{76.4} \mathrm{Te}_{16.5}$ amorphous film in a sealed quartz tube revealed that when the temperature was raised, this quaternary alloy maintained an A7-type structure, hardly showing any atomic shift. However, decomposition into two phases, $\mathrm{AgInTe}_{2}$ and Sb-Te, occurred at around $545 \mathrm{~K}$. After this decomposition, the atomic configuration of the $\mathrm{Sb}-\mathrm{Te}$ fragment with the A7-type disordered structure gradually moved into an ordered arrangement to finally obtain a stable homologous structure ruled by its composition. This can be considered as follows: the dopants, Ag and In, played roles in simplifying the structure of the quaternary alloy. However, once these dopants were lost, the structural feature of the SbTe binary compound revealed itself.

\section{Experimental}

A thin film of $\mathrm{Ag}_{3.4} \mathrm{In}_{3.7} \mathrm{Sb}_{76.4} \mathrm{Te}_{16.5}$ with a thickness of approximately $300 \mathrm{~nm}$ was formed by sputtering on a glass disk $120 \mathrm{~mm}$ in diameter. The film was scraped off with a spatula to create a powder, which was then packed into a quartz capillary tube with an internal diameter of $0.3 \mathrm{~mm}$. To prevent it from reacting with components of air, we sealed the opening of the capillary tube using an oxyacetylene flame. The

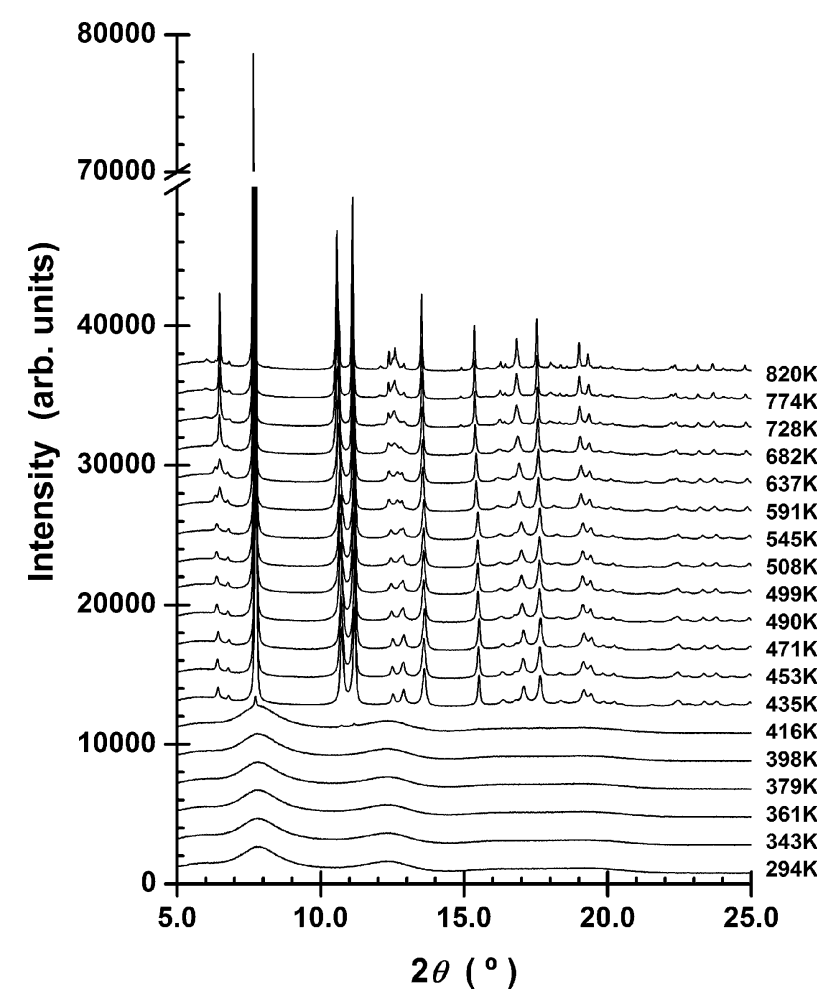

Figure 1

Temperature dependence of X-ray powder diffraction profiles for sputtered $\mathrm{Ag}_{3.4} \mathrm{In}_{3.7} \mathrm{Sb}_{76.4} \mathrm{Te}_{16.5}$ amorphous film in the heating process. The amorphous halo patterns are observed at low temperatures from 295 to $416 \mathrm{~K}$. However, at around $416 \mathrm{~K}$ the Bragg peaks of the A7-type structure appear in the halo pattern. As the temperature is raised further, the A7-type single phase separates into two phases, $\mathrm{AgInTe}_{2}$ and an SbTe binary compound, at around $545 \mathrm{~K}$. At $2 \theta$ angles lower than $5^{\circ}$, Bragg peaks were hardly observed at any measurement temperatures.
Table 1

Final structural parameters for $\mathrm{Ag}_{3.4} \mathrm{In}_{3.7} \mathrm{Sb}_{76.4} \mathrm{Te}_{16.5}$ at $545 \mathrm{~K}$ refined by Rietveld analysis.

The standard deviations are shown in parentheses. The diffraction data used for the analysis: $5.50 \leq 2 \theta \leq 31.50^{\circ}$. (a) Conventional three-dimensional Rietveld analysis results. The space group $R \overline{3} m$ was applied. (b) Results of a four-dimensional superspace analysis refined as a commensurately modulated structure, using the 'commensurate case' in the refinement program JANA; the modulation period $\gamma$ was maintained at $3 / 2$ during the refinements (the analysis assuming an incommensurate case also concluded that this crystal has a commensurate structure with $\gamma=3 / 2$ ). The superspace group was assumed to be $R \overline{3} m(00 \gamma) 00$ (de Wolff, 1974; de Wolff et al., 1981). $B_{1}^{s}$ represents the positional modulation amplitude. The determined $z$-slope (the form factor of the sawtooth function) value corresponds to $z=0.2359$ in the threedimensional description; this $z$ value shows good agreement with $z=0.2365$, which was derived from the three-dimensional analysis. $U^{i j}$ represent the atomic displacement parameters. It was assumed that the four atoms randomly occupy the atomic sites in both analyses.

(a) $R_{\mathrm{p}}=0.0215, R_{\mathrm{wp}}=0.0313, R F_{\text {obs }}=0.0157, R F_{\text {wobs }}=0.0238, a=4.3037$ (5) and $c=11.290$ (1) A. $M: \mathrm{Ag}_{0.034} \mathrm{In}_{0.037} \mathrm{Sb}_{0.764} \mathrm{Te}_{0.165}$.

\begin{tabular}{llllllll}
\hline Atom & Site & $g$ & $x$ & $y$ & $z$ & $U^{11}\left(\AA^{2}\right)$ & $U^{33}\left(\AA^{2}\right)$ \\
\hline$M$ & $6 c$ & 1.0 & 0 & 0 & $0.2365(1)$ & $0.0235(5)$ & $0.042(2)$ \\
\hline
\end{tabular}

(b) Commensurate case $\left(t_{0}=\frac{1}{4}, \frac{3}{4}\right)$.

$R$ factors of profile and all reflections

$\begin{array}{ll}R_{\mathrm{p}} & 0.0202 \\ R_{\mathrm{wp}} & 0.0292 \\ R F_{\text {obs }} & 0.0133 \\ R F_{\text {wobs }} & 0.0198\end{array}$

$R$ factors of main and satellite reflections

Main

$R F_{\text {obs }}$

0.0111

$R F_{\text {wobs }}$

0.0141

$R$ factors of satellites

First-order

$R F_{\text {obs }}$

0.0184

$R F_{\text {wobs }}$

0.0273

$\gamma=3 / 2 ; a=4.3037(5), c=5.6452(6) \AA$

\begin{tabular}{llllllll}
\hline Atom & $g$ & $x$ & $y$ & $z$ & $z$-slope & $U^{11}\left(\AA^{2}\right)$ & $U^{33}\left(\AA^{2}\right)$ \\
\hline$M$ & 1.0 & 0 & 0 & 0 & $-0.0563(8)$ & $0.0229(5)$ & $0.043(1)$ \\
\hline
\end{tabular}

diffraction experiments were carried out using the largediameter Debye-Scherrer camera with an imaging plate on the BL02B2 beamline at the Japan Synchrotron Radiation Research Institute (Nishibori et al., 2001). The energy of the incident beam was approximately $29.4 \mathrm{keV}$. An imaging plate with a pixel area of $100 \mu \mathrm{m}^{2}$ was used as the detector; this pixel area corresponded to an angular resolution of $0.02^{\circ}(287 \mathrm{~mm}$ camera diameter). However, for more precise structure analyses, intensity data in increments of $0.01^{\circ}$ were obtained by reading the imaging plate for a pixel area of $50 \mu^{2}$. Experiments at low and high temperatures were carried out while blowing nitrogen gas onto the capillary tube at the specified temperatures. The crystal structures were examined and refined using the Rietveld method (Rietveld, 1969); the programs JANA2000 (Petříček \& Dušek, 2000) and $J A N A 2006$ (Petrríček et al., 2006) were used for this purpose. The energy of the synchrotron radiation was confirmed by recording the diffraction intensity of $\mathrm{CeO}_{2}(a=5.4111 \AA)$ powder as a reference specimen at room temperature under 


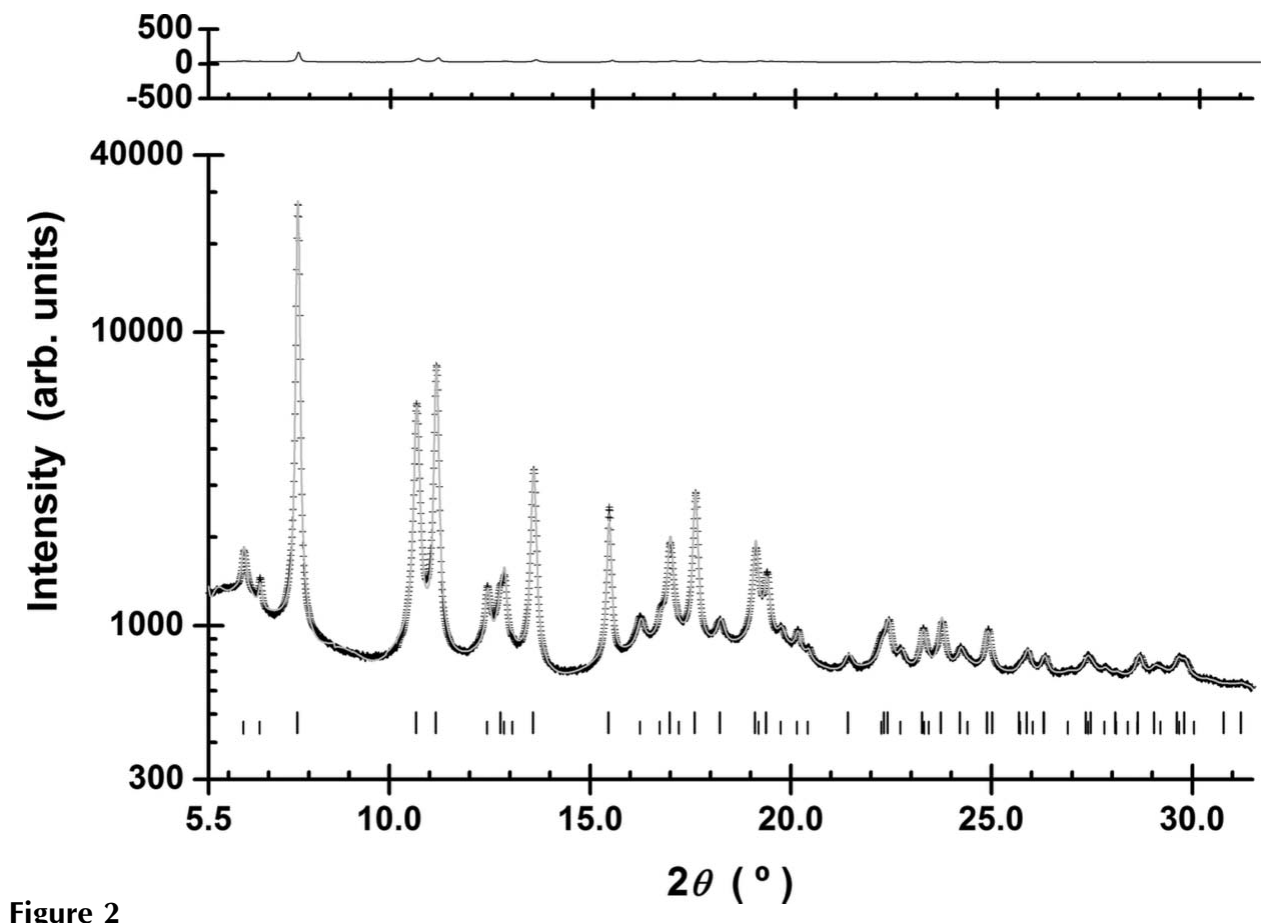

Observed (+) and calculated (gray line) X-ray diffraction profiles for $\mathrm{Ag}_{3.4} \mathrm{In}_{3.7} \mathrm{Sb}_{76.4} \mathrm{Te}_{16.5}$ (crystallized into an A7-type single structure) at $545 \mathrm{~K}$ by four-dimensional Rietveld analysis in the commensurate case. A difference curve (observed - calculated) appears at the top of the figure; reflection markers are indicated by vertical spikes below the diffraction patterns: the longer ones are for the peaks of the main reflections and the shorter ones for the satellites. the same conditions, which showed that the wavelength used for the structural analyses was 0.4187 (3) A. Neutral atomic scattering factors were employed for them.

\section{Results and discussion}

\subsection{Crystals observed in this experiment}

The diffraction patterns obtained for the sputtered $\mathrm{Ag}_{3.4} \mathrm{In}_{3.7} \mathrm{Sb}_{76.4} \mathrm{Te}_{16.5}$ amorphous film are shown in Fig. 1. The amorphous phase transformed into an A7-type crystalline single phase, as seen in this graph, at around $416 \mathrm{~K}$. The results of a search match and Rietveld analyses revealed the diffraction patterns taken from 435 to $545 \mathrm{~K}$ to be almost identical to that of an $\mathrm{As}, \mathrm{Sb}$ or $\mathrm{Bi}$ crystal with an A7-type structure (Clark, 1955), as has been previously elucidated (Matsunaga et al., 2001). The (conventional three-dimensional)

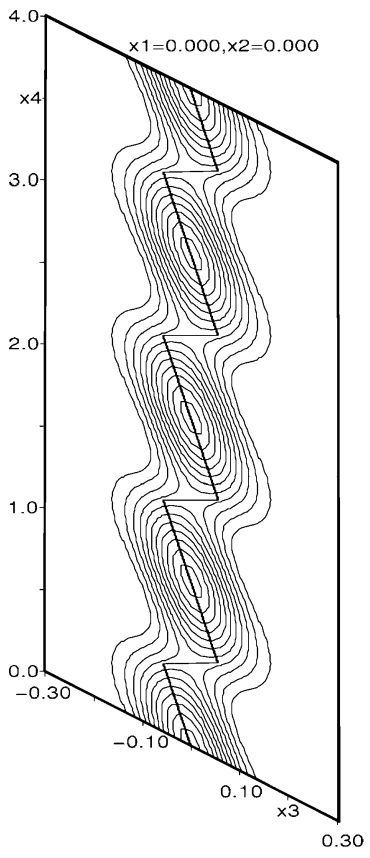

(a)

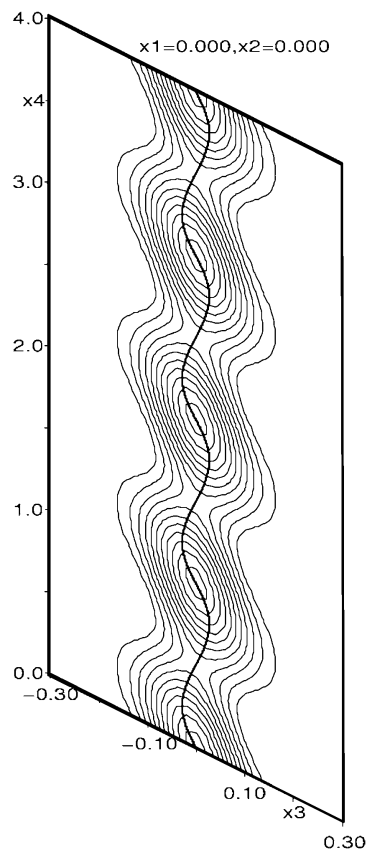

(b)
Figure 3

Electron-density maps for $\mathrm{Ag}_{3.4} \mathrm{In}_{3.7} \mathrm{Sb}_{76.4} \mathrm{Te}_{16.5}$ at $545 \mathrm{~K}$ depicted with $F_{\mathrm{o}}$; only positive contours are drawn at intervals of $200 \mathrm{e}^{-3}$. (a) The sawtooth-type modulation function used to describe the displacement of the atom in an asymmetric unit of this three-dimensional crystal is indicated by a solid line. As seen by comparison with map $(b)$ obtained from the Rietveld analysis with a harmonic function, analysis with the sawtooth function provided better results.
Rietveld analysis results at $545 \mathrm{~K}$ are shown in Table 1(a). This crystal belongs to the space group $R \overline{3} m$; the four constituent elements, $\mathrm{Ag}$, In, Sb and Te, randomly occupy the $6 c$ site (Matsunaga, Akola et al., 2011). The changes in the diffraction lines with increasing temperature show that the single-phase A7-type structure is maintained up to around $545 \mathrm{~K}$. However, the peaks for $\mathrm{CuFeS}_{2}$-type $\mathrm{AgInTe}_{2}$ (Wyckoff, 1986) appear at around $590 \mathrm{~K}$, along with those of the A7-type structure. This decomposition can be written as

$$
\mathrm{Ag}_{3.4} \mathrm{In}_{3.7} \mathrm{Sb}_{76.4} \mathrm{Te}_{16.5} \rightarrow \mathrm{Ag}_{3.4} \mathrm{In}_{3.4} \mathrm{Te}_{6.8}+\mathrm{In}_{0.3} \mathrm{Sb}_{76.4} \mathrm{Te}_{9.7}
$$

These two phases formed by heating coexisted up to the high temperatures at which their Bragg peaks almost disappeared as a result of dissolving. As seen in this equation, the second decomposition product can virtually be regarded as an $\mathrm{Sb}-\mathrm{Te}$ binary compound. Even at high temperatures close to the melting temperature, $\mathrm{AgInTe} \mathrm{T}_{2}$ tightly held the $\mathrm{CuFeS}_{2}$-type structure irrespective of temperature. However, our present analysis revealed that the structure of the second fragment, the $\mathrm{Sb}-\mathrm{Te}$ compound, gradually changed with increasing temperature until obtaining its final stable atomic configuration. These structures can be closely approximated by the A7type structure but are not real A7-type ones.

\subsection{Homologous structures}

We examined many types of chalcogenide materials to clarify the high-speed phase-change mechanism and develop 
Table 2

Space groups for $(\mathrm{GeTe})_{n}\left(\mathrm{Sb}_{2} \mathrm{Te}_{3}\right)_{m}$ pseudobinary compounds.

$N$ shows the number of atomic layers in the unit cell.

\begin{tabular}{lllll}
\hline Compound & $n$ & $m$ & $N$ & Space group \\
\hline $\mathrm{Ge}_{3} \mathrm{Sb}_{2} \mathrm{Te}_{6}$ & 3 & 1 & 33 & $R \overline{3} m$ \\
$\mathrm{Ge}_{2} \mathrm{Sb}_{2} \mathrm{Te}_{5}$ & 2 & 1 & 9 & $P \overline{3} m 1$ \\
$\mathrm{Ge}_{1} \mathrm{Sb}_{2} \mathrm{Te}_{4}$ & 1 & 1 & 21 & $R \overline{3} m$ \\
$\mathrm{Ge}_{1} \mathrm{Sb}_{4} \mathrm{Te}_{7}$ & 1 & 2 & 12 & $P \overline{3} m 1$ \\
$\mathrm{Ge}_{1} \mathrm{Sb}_{6} \mathrm{Te}_{10}$ & 1 & 3 & 51 & $R \overline{3} m$ \\
\hline
\end{tabular}

new materials for future ultra-high-density phase-change recording devices. This revealed that, after sufficient heat treatments, almost all these materials finally fell into their stable crystals with so-called homologous structures. It has been found that in thermal equilibrium, the typical phasechange materials, the GeTe- $\mathrm{Sb}_{2} \mathrm{Te}_{3}$ pseudobinary system, the $\mathrm{Sb}-\mathrm{Te}$ or $\mathrm{Bi}-\mathrm{Te}$ binary system, form various intermetallic compounds represented by the chemical formulae $(\mathrm{GeTe})_{n}\left(\mathrm{Sb}_{2} \mathrm{Te}_{3}\right)_{m},\left(\mathrm{Sb}_{2}\right)_{n}\left(\mathrm{Sb}_{2} \mathrm{Te}_{3}\right)_{m}$ or $\left(\mathrm{Bi}_{2}\right)_{n}\left(\mathrm{Bi}_{2} \mathrm{Te}_{3}\right)_{m}(n, m$ : integer). All these compounds have trigonal structures with $2 n$ $+5 m$ cubic close-packed periodicity (almost) without exception. [More specifically, the residual of $(2 n+5 m) / 3=0$ and $\neq 0$ leads to the formation of crystals having structures with primitive $(P)$ and rhombohedral $(R)$ unit cells; they form structures with $N=(2 n+5 m)$ and $N=3 *(2 n+5 m)$ layers, respectively.] Table 2 shows the case of the $\mathrm{GeTe}-\mathrm{Sb}_{2} \mathrm{Te}_{3}$ compounds; all of the existing intermetallic compounds in these systems follow this rule (Matsunaga \& Yamada, 2004a; Matsunaga, Yamada \& Kubota, 2004; Matsunaga et al., 2007a,b, 2010; Matsunaga, Kojima et al., 2008). This could also be confirmed from the relevant tables in other papers (Karpinsky et al., 1998; Kuznetsova et al., 2000; Shelimova et al., 2000, 2004; Shelimova, Karpinskii et al., 2001; Shelimova, Konstantinov et al., 2001; Poudeu \& Kanatzidis, 2005). These structures are similar to each other and systematically characterized by the stacking of the $(\mathrm{GeTe})_{n}$ and $\left(\mathrm{Sb}_{2} \mathrm{Te}_{3}\right)_{m},\left(\mathrm{Sb}_{2}\right)_{n}$ and $\left(\mathrm{Sb}_{2} \mathrm{Te}_{3}\right)_{m}$, or $\left(\mathrm{Bi}_{2}\right)_{n}$ and $\left(\mathrm{Bi}_{2} \mathrm{Te}_{3}\right)_{m}$ blocks along the $\mathbf{c}_{H}$ axes, with very long cell dimensions in the conventional threedimensional structure description (Karpinsky et al., 1998; Shelimova et al., 2000; Shelimova, Karpinskii et al., 2001; Poudeu \& Kanatzidis, 2005; Matsunaga \& Yamada, 2004a, Matsunaga, Yamada \& Kubota, 2004; Matsunaga et al., 2007a,b, 2010; Matsunaga, Kojima et al., 2008; Matsunaga, Morita et al., 2008). More generally and more precisely it has been assumed that these structures should be described as commensurately or incommensurately modulated fourdimensional structures characterized by modulation vectors $\mathbf{q}=\gamma \cdot \mathbf{c}^{*}$ (Lind \& Lidin, 2003), where $\gamma$ values are real numbers equal to or around $3(n+3 m) /(2 n+5 m)$ [see equation (3); $\mathbf{c}^{*}$ is the fundamental reciprocal vector formed by threelayer cubic stacking]. For instance, it has been clarified that, in the thermal equilibrium, $\operatorname{Sb}_{8} \mathrm{Te}_{3}(n=3$ and $m=1)$ has a homologous structure characterized by a modulation vector $\mathbf{q}=18 / 11 \cdot \mathbf{c}^{*}$ (Kifune et al., 2005, 2011). Thus, we applied this more universal four-dimensional superspace method for
Table 3

Final structural parameters.

(a) Final structural parameters determined by four-dimensional superspace refinements of $\mathrm{Sb}_{89} \mathrm{Te}_{11}$ and conventional three-dimensional refinements of $\mathrm{AgInTe}_{2}$ at room temperature. The $R$ factors for the entire pattern $\left(\mathrm{Sb}_{89} \mathrm{Te}_{11}+\right.$ AgInTe 2 ) are $R_{\mathrm{p}}=0.0205$ and $R_{\mathrm{wp}}=0.0297$. The superspace group for $\mathrm{Sb}_{89} \mathrm{Te}_{11}$ was assumed to be $R \overline{3} m(00 \gamma) 00$; on the other hand, $I \overline{4} 2 d$ was applied for $\mathrm{AgInTe}_{2}$. Both structures were refined simultaneously using a multiphase refinement. Diffraction data used for the analysis: $5.50 \leq 2 \theta \leq 42.10^{\circ}$. The standard deviations are shown in parentheses. $B_{n}^{s}$ represents the positional modulation amplitude, whereas $U \mathrm{~s}$ represent the atomic displacement parameters. (b) and (c) Final structural parameters obtained by fourdimensional superspace refinements of $\mathrm{Sb}_{89} \mathrm{Te}_{11}$ performed by applying sawtooth functions (Dušek et al., 2010) or harmonic functions orthogonalized to the crenel interval (Lind \& Lidin, 2003) instead of conventional harmonic functions. The $R$ factors for the entire pattern $\left(\mathrm{Sb}_{89} \mathrm{Te}_{11}+\mathrm{AgInTe}_{2}\right)$ are $R_{\mathrm{p}}=$ 0.0205 and $R_{\mathrm{wp}}=0.0297$, and $R_{\mathrm{p}}=0.0204$ and $R_{\mathrm{wp}}=0.0298$. $(d)$ The results obtained by the four-dimensional Rietveld analyses performed assuming that $\mathrm{Sb}_{89} \mathrm{Te}_{11}$ has a commensurately modulated structure; the modulation period $\gamma$ was maintained at $45 / 29$ during the refinements. The $R$ factors for the entire pattern are $R_{\mathrm{p}}=0.0202$ and $R_{\mathrm{wp}}=0.0295$. The superspace group was assumed to be the same as in the above incommensurate case. The electron density map and atomic positions obtained from this analysis in the commensurate case are not shown in this paper, but were almost identical to those seen in Fig. 6. Only the first- and second-order satellites were considered for these fourdimensional analyses ( $f=0,1$ and 2 were used for the analyses). This assumption gave sufficiently low $R$ values.

(a) $\mathrm{Sb}_{89} \mathrm{Te}_{11}$ :

$R$ factors of profile and all reflections

$$
\begin{array}{ll}
R F_{\text {obs }} & 0.0162 \\
R F_{\text {wobs }} & 0.0159
\end{array}
$$

$R$ factors of main and satellite reflections

$\begin{array}{lll}\text { Main } & R F_{\text {obs }} & 0.0144 \\ & R F_{\text {wobs }} & 0.0158\end{array}$

$R$ factors of satellites

First order

$R F_{\text {obs }} \quad 0.0186$

$R F_{\text {wobs }} \quad 0.0196$

Second order

$R F_{\text {obs }}$

0.0138

$R F_{\text {wobs }} \quad 0.0132$

$\gamma=1.5516(4) ; a=4.2969$ (1), $c=5.6759$ (2) ̊.

\begin{tabular}{lllllllll}
\hline Atom & $g$ & $x$ & $y$ & $z$ & $B_{1}^{s}$ & $B_{2}^{s}$ & $U^{11}\left(\AA^{2}\right)$ & $U^{33}\left(\AA^{2}\right)$ \\
$\mathrm{Sb}$ & 1.0 & 0 & 0 & 0 & $-0.0394(4)$ & $0.003(1)$ & $0.0123(2)$ & $0.0101(7)$ \\
$\mathrm{Te}$ & 1.0 & 0 & 0 & 0 & $-0.27(1)$ & - & 0.0123 & 0.0101 \\
\hline
\end{tabular}

$\mathrm{AgInTe}_{2}: R F_{\text {obs }}=0.0253, R F_{\text {wobs }}=0.0281, a=6.4275$ (3) and $c=12.6089$ (9) $⿱$.

\begin{tabular}{lllllll}
\hline Atom & Site & $g$ & $x$ & $y$ & $z$ & $U_{\text {iso }}\left(\AA^{2}\right)$ \\
\hline $\mathrm{Ag}$ & $4 b$ & $1 / 4$ & 0 & 0 & $1 / 2$ & 0.029 \\
$\mathrm{In}$ & $4 a$ & $1 / 4$ & 0 & 0 & 0 & 0.029 \\
$\mathrm{Te}$ & $8 d$ & $1 / 2$ & $0.252(11)$ & $1 / 4$ & $1 / 8$ & $0.029(1)$ \\
\hline
\end{tabular}

(b) $\mathrm{Sb}_{89} \mathrm{Te}_{11}$ :

$R$ factors of profile and all reflections

$\begin{array}{ll}R F_{\text {obs }} & 0.0168 \\ R F_{\text {wobs }} & 0.0176\end{array}$

$R$ factors of main and satellite reflections

Main

$R F_{\text {obs }} \quad 0.0123$

$R F_{\text {wobs }}$

0.0136

$R$ factors of satellites

First order

$R F_{\text {obs }}$

0.0221

$R F_{\text {wobs }} \quad 0.0238$ 
Table 3 (continued)

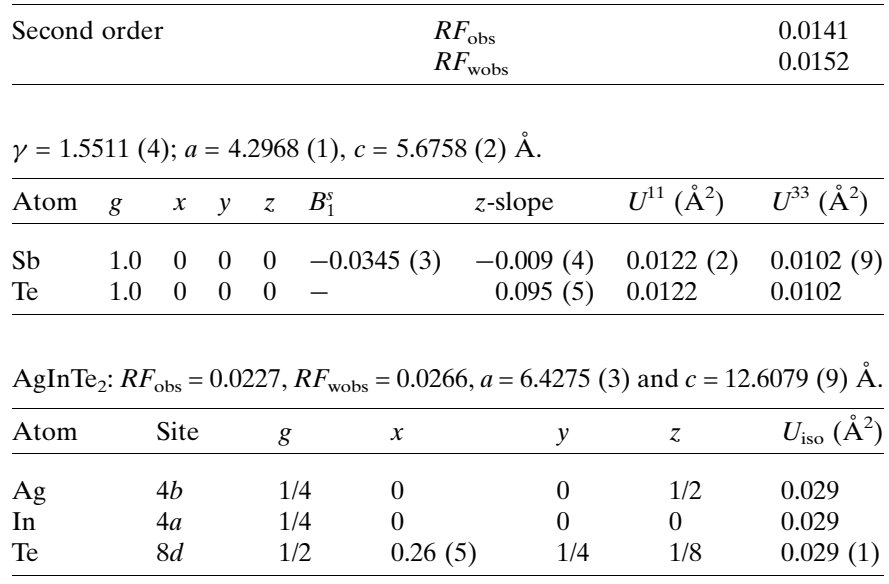

(c) $\mathrm{Sb}_{89} \mathrm{Te}_{11}$ :

$R$ factors of profile and all reflections $\begin{array}{ll}R F_{\text {obs }} & 0.0156 \\ R F_{\text {wos }} & 0.0164\end{array}$

$R$ factors of main and satellite reflections

$\begin{array}{lll}\text { Main } & R F_{\text {obs }} & 0.0115 \\ R F_{\text {wobs }} & 0.0128\end{array}$

$R$ factors of satellites

\begin{tabular}{lll} 
First order & $R F_{\text {obs }}$ & 0.0202 \\
& $R F_{\text {wobs }}$ & 0.0218 \\
Secnd order & $R F_{\text {obs }}$ & \\
& $R F_{\text {wobs }}$ & 0.0141 \\
& & 0.0142 \\
\hline
\end{tabular}

$\gamma=1.5511$ (4); $a=4.2968$ (1), $c=5.6759$ (2) Å.

\begin{tabular}{lllllllll}
\hline Atom & $g$ & $x$ & $y$ & $z$ & Zort1 & Zort3 & $U^{11}\left(\AA^{2}\right)$ & $U^{33}\left(\AA^{2}\right)$ \\
\hline $\mathrm{Sb}$ & 1.0 & 0 & 0 & 0 & $-0.0314(3)$ & $-0.001(1)$ & $0.0122(2)$ & $0.0102(8)$ \\
$\mathrm{Te}$ & 1.0 & 0 & 0 & 0 & $-0.049(3)$ & - & 0.0122 & 0.0102 \\
\hline
\end{tabular}

$\mathrm{AgInTe}_{2}: R F_{\text {obs }}=0.0227, R F_{\text {wobs }}=0.0257, a=6.4274$ (3) and $c=12.6084$ (9) $\AA$.

\begin{tabular}{lllllll}
\hline Atom & Site & $g$ & $x$ & $y$ & $z$ & $U_{\text {iso }}\left(\AA^{2}\right)$ \\
\hline $\mathrm{Ag}$ & $4 b$ & $1 / 4$ & 0 & 0 & $1 / 2$ & 0.028 \\
$\mathrm{In}$ & $4 a$ & $1 / 4$ & 0 & 0 & 0 & 0.028 \\
$\mathrm{Te}$ & $8 d$ & $1 / 2$ & $0.255(6)$ & $1 / 4$ & $1 / 8$ & $0.028(1)$ \\
\hline
\end{tabular}

(d) Commensurate case $\left(t_{0}=\frac{1}{50}+\frac{n}{25} ; n=0,1,2, \ldots\right) . \mathrm{Sb}_{89} \mathrm{Te}_{11}$ :

$R$ factors of profile and all reflections

$$
R F_{\text {obs }}
$$$$
R F_{\text {wobs }}
$$

0.0158 0.0159

$R$ factors of main and satellite reflections

Main

$$
\begin{aligned}
& R F_{\text {obs }} \\
& R F_{\text {wobs }}
\end{aligned}
$$

0.0135

0.0150

$R$ factors of satellites

\begin{tabular}{lll} 
First order & $R F_{\text {obs }}$ & 0.0182 \\
& $R F_{\text {wobs }}$ & 0.0197 \\
& & \\
Second order & $R F_{\text {obs }}$ & 0.0160 \\
& $R F_{\text {wobs }}$ & 0.0137 \\
\hline
\end{tabular}

$\gamma=45 / 29 ; a=4.2968$ (1), $c=5.6758$ (2) ̊.
Table 3 (continued)

\begin{tabular}{lllllllll}
\hline Atom & $g$ & $x$ & $y$ & $z$ & $B_{1}^{s}$ & $B_{2}^{s}$ & $U^{11}\left(\AA^{2}\right)$ & $U^{33}\left(\AA^{2}\right)$ \\
\hline $\mathrm{Sb}$ & 1 & 0 & 0 & 0 & $-0.0401(4)$ & $0.001(1)$ & $0.0119(2)$ & $0.0107(9)$ \\
$\mathrm{Te}$ & 1 & 0 & 0 & 0 & $-0.31(2)$ & - & 0.0119 & 0.0107 \\
\hline
\end{tabular}

$\mathrm{AgInTe}_{2}: R F_{\text {obs }}=0.0241, R F_{\text {wobs }}=0.0273, a=6.4276$ (3) and $c=12.6082$ (9) $\AA$.

\begin{tabular}{lllllll}
\hline Atom & Site & $g$ & $x$ & $y$ & $z$ & $U_{\text {iso }}\left(\AA^{2}\right)$ \\
\hline $\mathrm{Ag}$ & $4 b$ & 1.0 & 0 & 0 & $1 / 2$ & 0.028 \\
$\mathrm{In}$ & $4 a$ & 1.0 & 0 & 0 & 0 & 0.028 \\
$\mathrm{Te}$ & $8 d$ & 1.0 & $0.254(7)$ & $1 / 4$ & $1 / 8$ & $0.028(1)$ \\
\hline
\end{tabular}

analysis of the Sb-Te compound formed by thermal decomposition [see equation (1)].

\subsection{Structures of $A g_{3.4} \ln _{3.7} S b_{76.4} T_{16.5}$ and $\mathrm{Sb}_{89} \mathrm{Te}_{11}$ compounds}

As the initial structure models for the four-dimensional Rietveld refinements we adopted the layer stacking structures defined by the respective $\gamma$ values. In other words, in the SbTe compounds examined in this study, the modulation functions for $\mathrm{Sb}$ and $\mathrm{Te}$ atoms were respectively distributed around $t=0$ and $t=1 / 2\left(t\right.$ : internal parameter along the $x_{4}$ axis, the fourth crystal axis in four-dimensional space; Lind \& Lidin, 2003). This corresponds to a structure in which $\mathrm{Sb}$ and $\mathrm{Te}$ are placed at $0,0,0$ and their atomic species are distinguished using crenel functions (for $\mathrm{Sb}_{89} \mathrm{Te}_{11}$, width: 0.89 + center: 0 for Sb and width: $0.11+$ center: 0.5 for Te). As there is a difference of only one between the atomic numbers of $\mathrm{Sb}^{51}$ and $\mathrm{Te}^{52}$, it is very difficult for us to distinguish the kinds of atoms in their unit cells. We use the assumption that all of the Sb-Te crystals examined in this study have perfectly ordered atomic arrangements like those of other (binary) systems. The intensities of the satellites for $\mathrm{Sb}-\mathrm{Te}$ compounds are rather weak in general. Those of $\mathrm{Sb}_{89} \mathrm{Te}_{11}$ are no exception; almost all of the satellites observed were reproduced by adopting the maximum satellite index of 2 for the Rietveld analyses. The atomic displacements were represented using harmonic functions.

The four-dimensional Rietveld analyses performed with the diffraction patterns in Fig. 1, as mentioned above, provided the structural dependence on the temperature for $\mathrm{Ag}_{3.4} \mathrm{In}_{3.7} \mathrm{Sb}_{76.4} \mathrm{Te}_{16.5}$ (at low temperatures) and its thermally decomposed materials (at high temperatures). The results of the Rietveld analyses at $545 \mathrm{~K}$ for $\mathrm{Ag}_{3.4} \mathrm{In}_{3.7} \mathrm{Sb}_{76.4} \mathrm{Te}_{16.5}$, whose crystal still maintains an A7-type structure, are shown in Table $1(b)$ and Fig. 2 (cf. Table $1 a$, from the three-dimensional Rietveld analysis). In the four-dimensional analysis for this crystal, displacement for only a single atom in a threedimensional asymmetric unit has to be described by selecting the appropriate one from among several kinds of modulation functions; in this case, the use of a sawtooth function (Dušek et al., 2010) was revealed to give better results than a harmonic function, as seen in Fourier maps based on $\boldsymbol{F}_{\mathrm{o}}$ (Fig. 3). We can see from Fig. 4, $\gamma$ maintained a constant value of 1.5 up to a 
temperature of around $590 \mathrm{~K}$, at which $\mathrm{AgInTe}_{2}$ came out. However, above this temperature, $\gamma$ grew larger with increasing temperature and reached a value of around 1.55 at high temperatures near the melting point of $\mathrm{Sb}_{76.4} \mathrm{Te}_{9.7}$ (= $\mathrm{Sb}_{88.7} \mathrm{Te}_{11.3}$ when expressed as a percentage), which was found at around $870 \mathrm{~K}$ according to our present hightemperature measurement. The $\gamma$ value was maintained at around 1.55 even when the powder specimen was cooled back to room temperature. The results of the Rietveld analysis at room temperature are shown in Table $3(a)$ and Fig. 5. The refined modulation functions of $\mathrm{Sb}$ and $\mathrm{Te}$ and the corresponding de Wolff section of the observed Fourier map are shown in Fig. 6(a), together with a Fourier map based on $F_{\mathrm{o}}$. The difference Fourier maps obtained from the determined structure models exhibited few significant residual peaks, which showed the need for further structural modification or improvement. This was similar to those obtained at the high temperatures of 774 and $820 \mathrm{~K}$. The $\gamma$ values of around 1.55 found in these stagnated structures correspond well with the value of 1.5565 expected from the composition of $\mathrm{Sb}_{88.7} \mathrm{Te}_{11.3}$. Here we can simply derive $\gamma$ in terms of $x$ as

$$
\gamma=2-x / 2
$$

when the chemical formula for the binary system is written as $\mathrm{Sb}_{x} \mathrm{Te}_{1-x}$ or $\mathrm{Bi}_{x} \mathrm{Te}_{1-x}$ (Lind \& Lidin, 2003). It can be considered that after $\mathrm{Sb}_{76.4} \mathrm{Te}_{9.7}$ was segregated from $\mathrm{AgInTe}{ }_{2}$, it revealed its original crystalline nature to change the layer period $(\gamma)$ from $1.5(n=1, m=0$; A7-type six-layer structure) to 1.55 (another long-period layer structure), and it also clarified that, surprisingly enough, even an $\mathrm{Sb}-\mathrm{Te}$ compound with as much as $89 \%$ Sb can exist as a single homologous structure in its binary system. We can obtain

$$
\gamma=3(n+3 m) /(2 n+5 m) \text { and } x=2(n+m) /(2 n+5 m) \text {, }
$$

by comparing $\mathrm{Sb}_{x} \mathrm{Te}_{1-x}$ with $\left(\mathrm{Sb}_{2}\right)_{n}\left(\mathrm{Sb}_{2} \mathrm{Te}_{3}\right)_{m}$. The crystal structure $(\gamma \simeq 1.55)$ at high temperatures at which the $\gamma$ growth became stagnant can be approximated reasonably well by a commensurately modulated 29-layer structure with $n=12$ and $m=1(\gamma=45 / 29$; this three-dimensional structure model is shown in Fig. 7a). We also carried out a Rietveld analysis assuming that this $\mathrm{Sb}-\mathrm{Te}$ crystal had a commensurately modulated structure with this rational number of $\gamma$. As shown in Table $3(d)$, this analysis gave almost the same good results as in the incommensurate case (however, for this material it could not be concluded that it had transformed to a commensurate three-dimensional structure, in contrast to $\mathrm{Sb}_{87} \mathrm{Te}_{13}$, which will be discussed later). The Fourier map obtained from this four-dimensional analysis performed in the commensurate case is almost identical with all those in Fig. 6, as expected. In addition to the $\gamma$ dependence on temperature of this $\mathrm{Sb}-\mathrm{Te}$ material, the $\mathrm{Sb}_{8} \mathrm{Te}_{3}$ sputtered amorphous film showed a small $\gamma$ value just after the transformation to the crystalline phase. However, $\gamma$ became larger with increasing temperature to finally obtain its original long-period layer structure. We found that, in addition to the $\mathrm{Sb}_{8} \mathrm{Te}_{3}$ film, some other $\mathrm{Sb}-\mathrm{Te}$ films at various compositions show very similar behavior (we will show the results for these materials elsewhere in the near future). These results strongly suggest that not a few (at least) Sb-Te compounds, just after their crystal formations, transiently assume small $\gamma$ values (it is highly probable that these $\gamma$ values all start at 3/2), and when adequately treated with heat, they become larger to attain their respective, intrinsic homologous structures, depending on their binary compositions.

The atomic displacements (modulation functions) have already been shown in a stagnant structure of $\mathrm{Sb}_{89} \mathrm{Te}_{11}$ (see Fig. 6). However, just after the phase decomposition, the atomic displacements varied appreciably, as seen in Fig. 8. This figure shows, however, that such a varied atomic arrangement becomes more moderate with increasing temperature, and comes closer to those observed in the stagnated structures. In response to this structural change, although the interatomic distances in $\mathrm{Sb}_{89} \mathrm{Te}_{11}$ just after the phase decomposition are rather dispersed, especially for $\mathrm{Te}-\mathrm{Sb}$ pairs, they converge with the structural inactivation, as seen in Fig. 9. As mentioned above, before the phase decomposition, $\mathrm{Sb}$ and Te atoms (and the dopants) were randomly distributed in the A7-type structure. Therefore, it is expected that just after the decomposition, the crystal still has a strongly disordered atomic arrangement. However, during the structural change with increasing temperature, it gradually attains the perfectly ordered structure shown above (Kifune et al., 2011).

As mentioned above, we used harmonic functions to describe the atomic displacements. However, to examine them more precisely, the $J A N A 2006$ program provides several other functions in addition to the harmonic one. We performed further analyses by using some of these functions. However, these analyses gave us almost the same results as shown in Fig. 6 and Tables $3(b)$ and $(c)$.

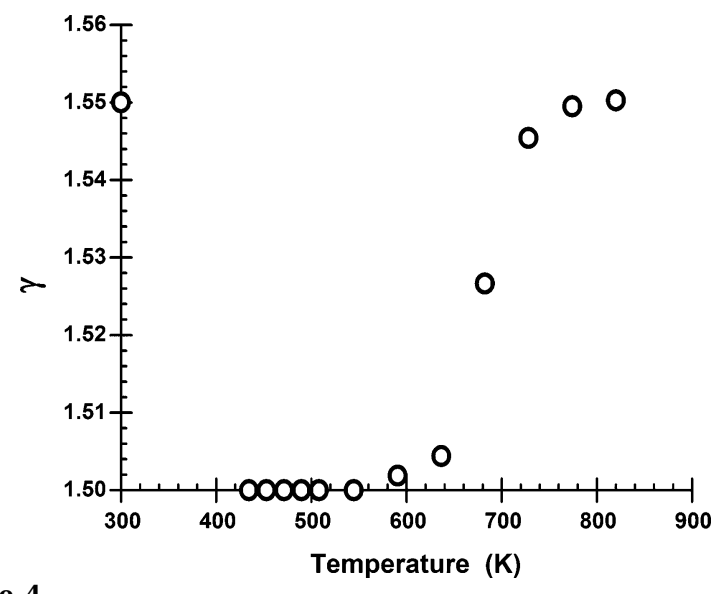

Figure 4

Temperature dependences of modulation period $\gamma$ obtained from fourdimensional structural analyses. The diffraction measurements were first made in the heating process up to $820 \mathrm{~K}$; then the powder specimen was cooled down for a room-temperature measurement. Error bars were omitted because they were smaller than the symbols shown. 


\subsection{Suitability of Sb-Te-based compounds for high-speed phase-change recording devices}

As has so far been shown, an Sb-Te compound containing $\mathrm{Ag}$ and In maintains a six-layer structure $(\gamma=3 / 2)$ up to a high temperature, at which phase separation occurs. On the other

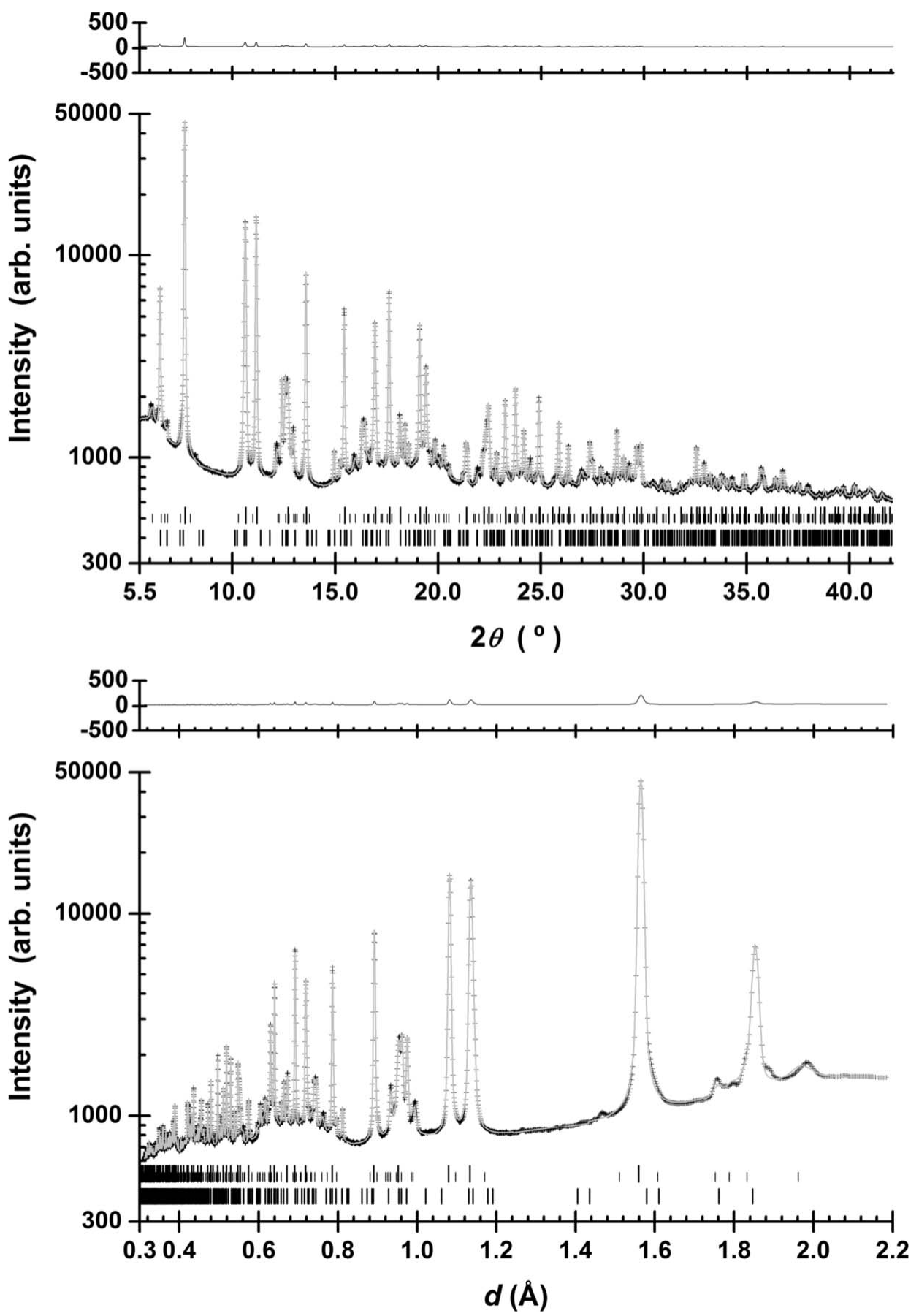

Figure 5

Observed (+) and calculated (gray line) X-ray diffraction profiles for $A g I n T e_{2}+S_{89} \mathrm{Te}_{11}$ at room temperature by Rietveld analysis. A difference curve (observed-calculated) appears at the top of the figure; reflection markers are indicated by vertical spikes below the diffraction patterns. Of the first line of spikes, the longer ones are for the peaks of the main reflections of $\mathrm{Sb}_{89} \mathrm{Te}_{11}$, and the shorter ones are for the satellites. Those at the bottom show the peak positions for $\mathrm{AgInTe}_{2}$. As seen in the figure depicted in $d$ spacing, a few unmatched weak peaks were observed, which were presumed to be from another contaminant phase. hand, Sb-Te films without such dopants show $\gamma$ values larger than

$3 / 2$ immediately after the crystallization. This strongly suggests that the $\mathrm{Ag}$ and In dopants play roles in maintaining the simple structure of the Sb-Te matrix. It also inversely implies that every $\mathrm{Sb}-\mathrm{Te}$ binary-compound film will experience a sixlayer structure in a very short time right after the crystal formation because six layers is the simplest and shortest layer structure out of all of the possible homologous structures from $\mathrm{Sb}(\gamma=3 / 2)$ to $\mathrm{Sb}_{2} \mathrm{Te}_{3}(\gamma=9 / 5)$. Many studies (for instance, Matsunaga, Akola et al., 2011) have shown that phasechange chalcogenide amorphous materials have spatially isotropic atomic arrangements; it is highly likely that they crystallize once into simple and spatially isotropic structures, like a cubic crystal, because a six-layer (A7-type) structure can be well approximated by simple cubic lattices (Matsunaga \& Yamada, 2004b).

The atomic configuration in the amorphous phase of this material, which has already been revealed (Matsunaga, Akola et al., 2011), is highly disordered, similar to that of a liquid, and spatially has a completely isotropic symmetry. However, it has also been revealed that it has $3+3$ coordination structures even in such a disordered atomic arrangement, as well as that of the crystalline phase: that is, both phases have very similar coordination structures, i.e. locally very similar atomic arrangements (it is well known that an A7-type crystal has a $3+3$ coordination structure; Clark, 1955; Hoffmann, 1988). This is one of the major reasons that this material achieves a sufficiently high phase-change speed by locally minimal bond interchanges. As for the dopants, it has been presumed that either or both Ag and In atoms probably raise the crystallization temperature of the amorphous phase to obtain a sufficient endurance for long-term data preservation. In addition, as mentioned above, they make the atomic arrangement of the crystal simple and spatially isotropic, holding the 
Table 4

Final structural parameters and $R$ factors.

(a) Final structural parameters for $\mathrm{Ag}_{3.4} \mathrm{In}_{3.7} \mathrm{Sb}_{76.4} \mathrm{Te}_{16.5}$ refined using conventional three-dimensional Rietveld analyses. In these analyses it was assumed that this material kept an unchanged A7-type structure while $\mathrm{AgInTe}_{2}$ formed at around $590 \mathrm{~K}$ with increasing temperature. The space group $R \overline{3} m$ was applied to all structure models, despite the fact that for $z=1 / 4$ $R 3 m$ or $R 32$ should be used instead (Matsunaga et al., 2001). The standard deviations are shown in parentheses, and the reliability factors are indicated as percentages. (b) The $R$-factors (shown as percentages) obtained through the four-dimensional analyses (see Table $5 a$ ) are also tabulated for comparison.

\begin{tabular}{|c|c|c|c|c|c|c|c|}
\hline \multicolumn{6}{|l|}{ (a) } & \multicolumn{2}{|l|}{ (b) } \\
\hline$T(\mathrm{~K})$ & $a(\AA)$ & $c(\AA)$ & $z$ & $R F_{\text {obs }}$ & $R F_{\text {wobs }}$ & $R F_{\text {obs }}$ & $R F_{\text {wobs }}$ \\
\hline 435 & $4.3010(6)$ & $11.202(2)$ & $0.2356(1)$ & 1.37 & 1.83 & & \\
\hline 453 & 4.3013 (6) & $11.212(2)$ & 0.2359 (1) & 1.47 & 2.15 & & \\
\hline 471 & $4.3015(6)$ & $11.223(2)$ & $0.2360(1)$ & 1.90 & 2.15 & & \\
\hline 490 & 4.3019 (6) & $11.242(2)$ & $0.2362(1)$ & 2.06 & 2.35 & & \\
\hline 508 & $4.3033(6)$ & $11.267(2)$ & $0.2364(1)$ & 1.73 & 2.33 & & \\
\hline 545 & $4.3037(5)$ & $11.290(1)$ & $0.2365(1)$ & 1.57 & 2.38 & & \\
\hline 591 & $4.3084(1)$ & $11.330(1)$ & $0.2365(1)$ & 1.80 & 2.36 & 1.71 & 1.82 \\
\hline 637 & $4.3094(3)$ & 11.357 (1) & $0.2373(1)$ & 1.84 & 2.02 & 1.49 & 1.71 \\
\hline 682 & $4.3110(4)$ & $11.399(1)$ & $0.2400(1)$ & 2.37 & 2.79 & 1.23 & 1.29 \\
\hline 728 & $4.3127(3)$ & $11.438(1)$ & $0.2460(4)$ & 2.65 & 3.60 & 1.43 & 1.45 \\
\hline 774 & 4.3159 (3) & 11.439 (1) & 0.25 & 2.25 & 2.14 & 1.60 & 1.74 \\
\hline 820 & $4.3202(3)$ & 11.429 (1) & 0.25 & 1.94 & 1.81 & 1.75 & 1.78 \\
\hline
\end{tabular}

material in a single phase. It is expected that these are indispensable features for high-speed rewritable data storage media. In the near future, however, the individual roles played by $\mathrm{Ag}$ and $\mathrm{In}$ in the phase change of this material should be clarified.

\subsection{Three- or six-layer structure approximation for $\mathrm{Sb}-\mathrm{Te}$ compounds}

In our previous work (Matsunaga et al., 2001) Sb-Te compounds with small amounts of $\mathrm{Ag}$ or In were concluded to hold an A7-type structure up to the melting point. However, we must say that these compounds do not have an exact A7type structure but a long-period modulated structure defined by the binary composition in thermal equilibrium. The A7type (six-layer) structure is also one of the modulated structures (corresponding to the shortest period one). All the above-mentioned modulated structures can be approximated by a (cubic stacked) three-layer structure, which provides the fundamental lines in the diffraction patterns. If atoms at the $6 c$ site are located at $z=1 / 4$ in the A7-type structure, it corresponds to the three-layer structure. In the previous temperature measurement, one end of the capillary holding the powder specimen was open to the air, which yielded not a little $\mathrm{Sb}$

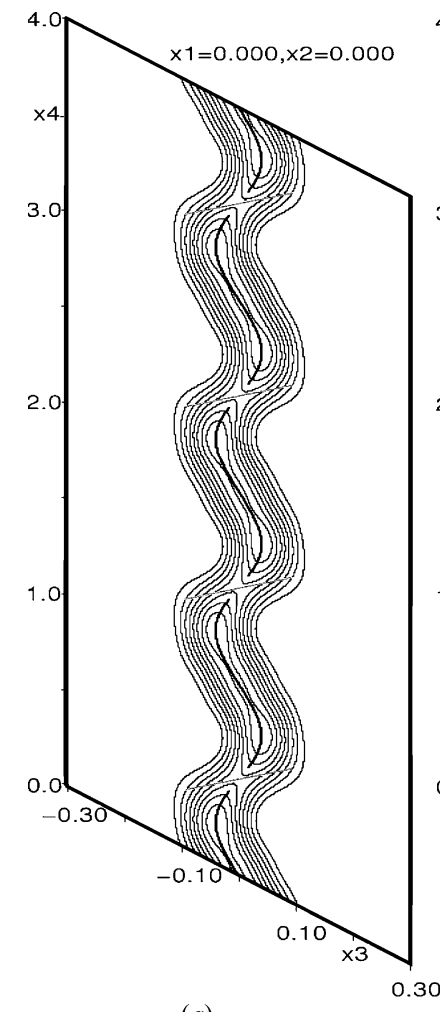

(a)

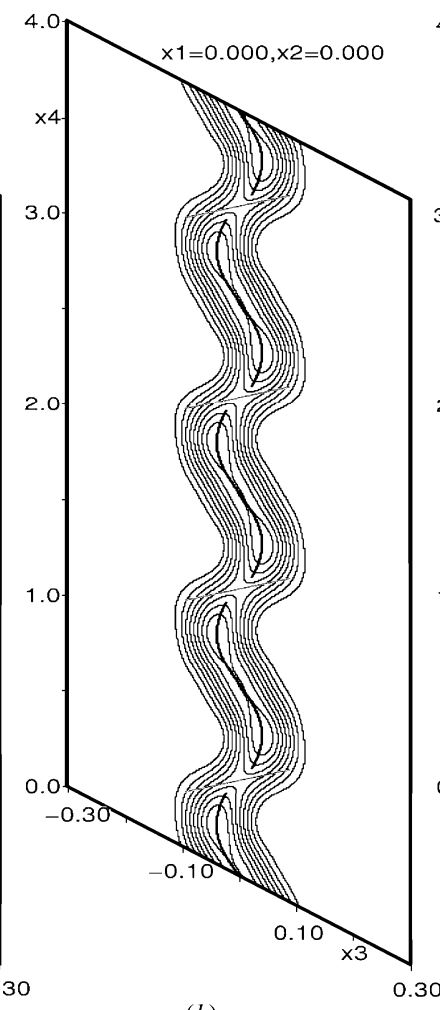

(b)

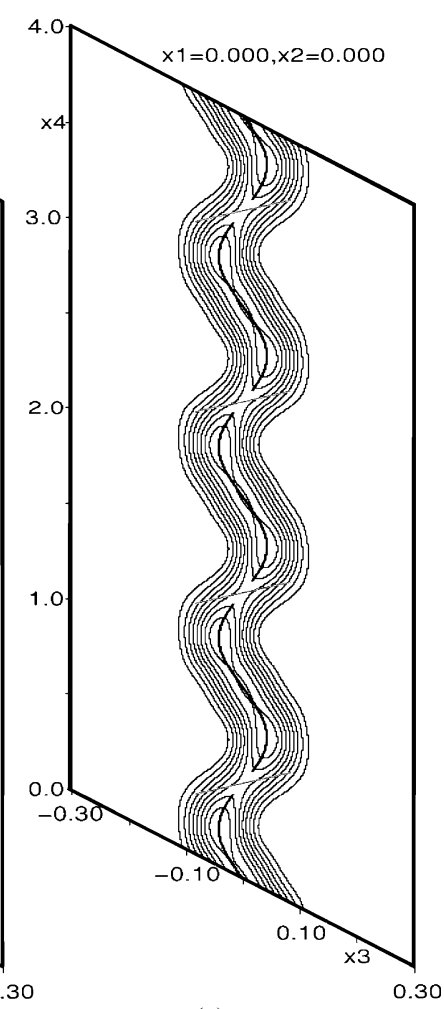

(c)
Figure 6

Electron-density maps for $\mathrm{Sb}_{89} \mathrm{Te}_{11}$ at room temperature based on $F_{\mathrm{o}}$; only positive contours are drawn at intervals of $500 \mathrm{e} \mathrm{A}^{-3}$. These maps, $(a),(b)$ and $(c)$, correspond to the results obtained from three kinds of the Rietveld analyses, $(a),(b)$ and $(c)$, shown in Table 3 . The crystal obtained a stagnant atomic arrangement after high-temperature measurements. These maps are almost identical to Fourier maps based on $F_{\mathrm{c}}$. The modulations in the displacement of the two atoms in this binary alloy are indicated by the crosslying curves for $\mathrm{Sb}$ in black and for Te in gray. These were analyzed by using different functions to describe the atomic displacement; however, we can see that they are almost the same. oxide (Fig. 10). This oxide formed a line of unnecessary Bragg peaks, which hindered us from determining the (weak) satellite peaks identifying the layer period of the structure. Further, at that time, such modulated structures were not familiarly associated with the Sb-Te binary system. All these factors made it difficult for us to discern that these Sb-Tebased alloys can take modulated structures. Thus, in previous work the A7-type structure was exclusively applied in the structural analyses, irrespective of the measurement temperature, which provided apparently sufficient results. In addition, in this work the same structural analysis was carried out to confirm the reproducibility of the previous work; we analyzed the structures by applying this simple $6 R$ structure to them. The results are shown in Table 4. As shown in this table, the $R$ factors were sufficiently low and the positional parameter $z$ gradually increased with temperature (which meant that the structure model for the Rietveld analysis gradually became 


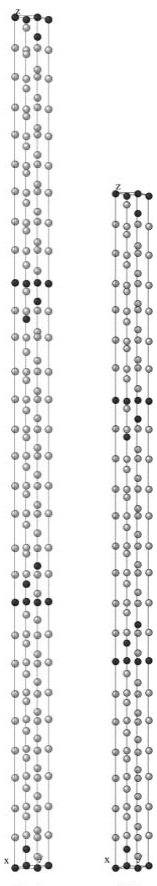

(a) (b)

Figure 7

Structural models of $(a) \mathrm{Sb}_{26} \mathrm{Te}_{3}$ and $(b) \mathrm{Sb}_{20} \mathrm{Te}_{3}$. The atomic positions of $\mathrm{Sb}$ and $\mathrm{Te}$ are shown by gray and black, respectively.

closer to the three-layer structure), which accurately reproduced the previous results. However, the agreement between the profiles of the observed and calculated intensities became worse as the temperature rose, especially for the (weak)

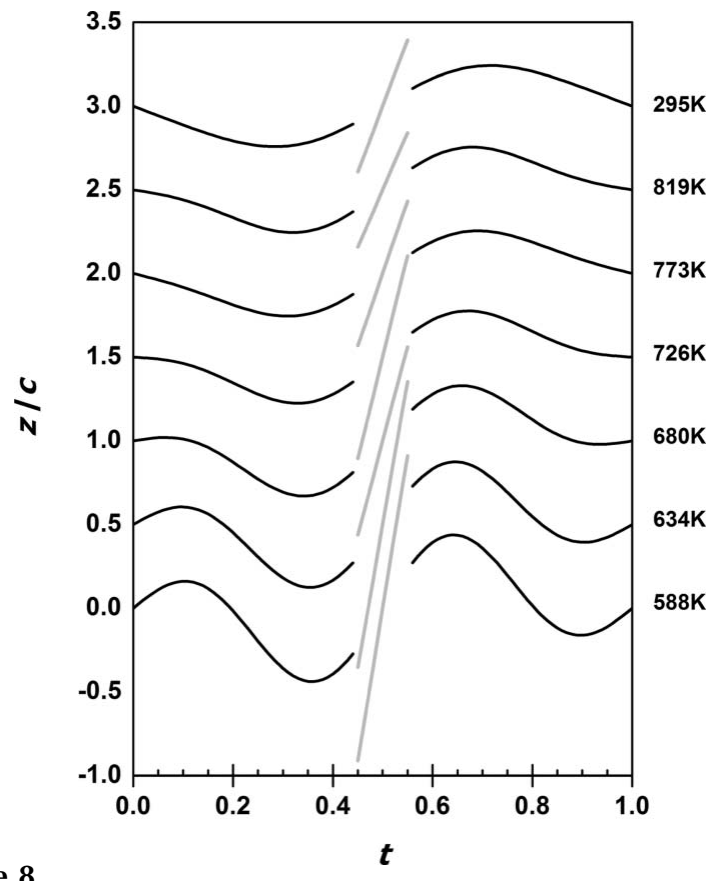

Figure 8

Displacements of $z$ as a function of $t$. Each profile is shifted by $d z=0.5$ with respect to the previous one (horizontal lines correspond to $d z=0$ for each group of profiles). The centers of modulation functions of $\mathrm{Sb}$ and Te atoms are located at the centers of $t=0$ and $1 / 2$, which are shown in black and gray, respectively. satellite reflections. On the other hand, those obtained through the four-dimensional analyses showed good agreement with each other, even at high temperatures near the melting point, as seen in Fig. 10. This strongly indicates that at high temperatures beyond the phase separation (or in the thermal equilibrium), this Sb-Te compound is not an A7-type structure $(\gamma=3 / 2)$ itself but one of the homologous structures defined by $\gamma>3 / 2$.

The present examination clarified that this $\mathrm{Sb}-\mathrm{Te}$ compound has a long-period modulated structure like that of the aforementioned $\mathrm{Sb}_{89} \mathrm{Te}_{11}$. The modulation period $\gamma$ kept a constant value of almost 1.5 up to a temperature of around $600 \mathrm{~K}$, at which the oxidation of Sb started. However, above this temperature, just as in the $\mathrm{Sb}_{89} \mathrm{Te}_{11}$ case, $\gamma$ grew larger with increasing temperature and reached a value of around 1.56 at high temperatures near the melting point (in contrast, in the previous experiment, Bragg peaks corresponding to $\mathrm{AgInTe}_{2}$ were hardly observed for some reason). This $\gamma$ value indicated that the composition of the compound should be $c a$ $\mathrm{Sb}_{87} \mathrm{Te}_{13}$ [see equation (2)]. Here, we ignore the locations of $\mathrm{Ag}$ and In because they are minor elements. We carried out

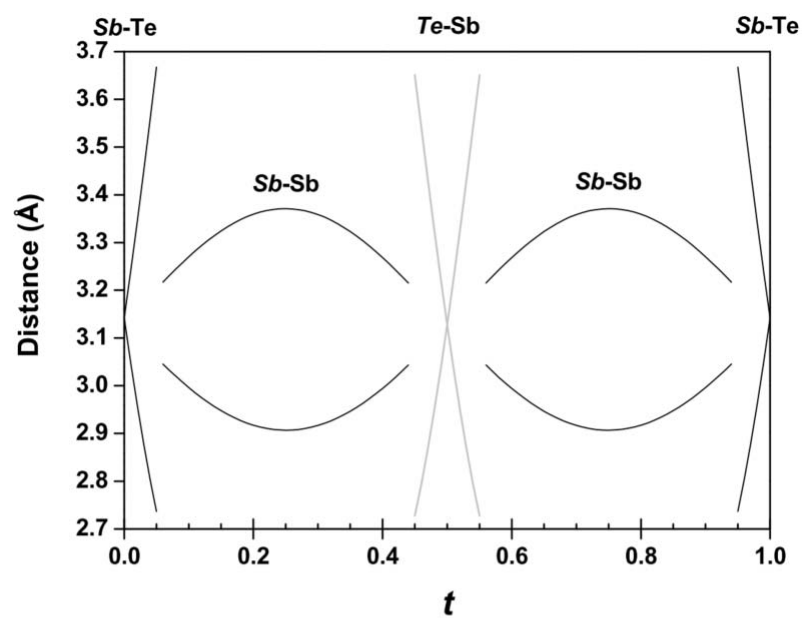

(a)

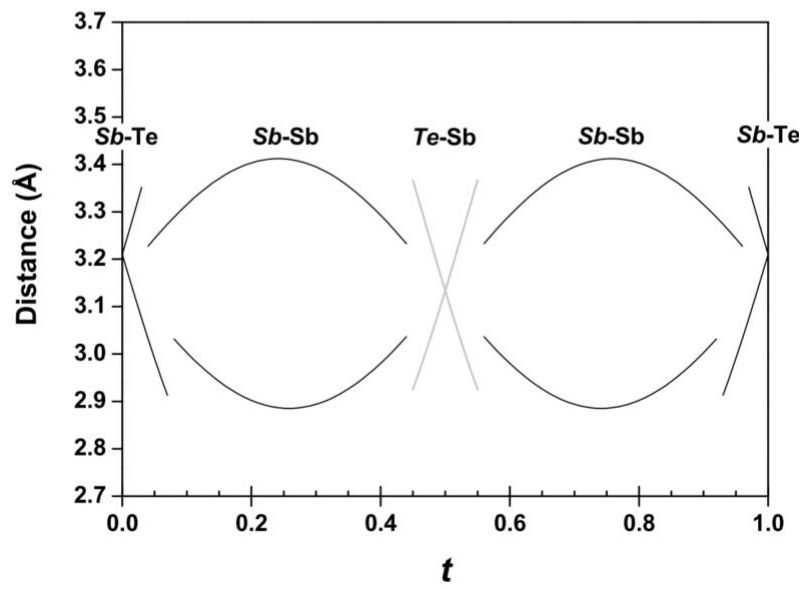

(b)

Figure 9

Interatomic distances versus $t$ for $\mathrm{Sb}_{89} \mathrm{Te}_{11}:(a)$ at $820 \mathrm{~K}$ near the melting temperature and $(b)$ at $637 \mathrm{~K}$ just after phase decomposition. The central atoms are shown in italics. 
Table 5

Final $R$ factors for $\mathrm{Sb}_{87} \mathrm{Te}_{13}$ at $773 \mathrm{~K}$ obtained through four-dimensional Rietveld analyses performed assuming that it has $(a)$ an incommensurately modulated structure ( $R$ factors for the entire pattern are $R_{\mathrm{p}}=$ 0.0201 and $\left.R_{\mathrm{wp}}=0.0294\right)$ and (b) a commensurately modulated structure.

The modulation period, $\gamma$, was maintained at $36 / 23$ during the refinements. The $R$ factors for the entire pattern are: $(b 1) R_{\mathrm{wp}}=0.0201$ and $R_{\mathrm{p}}=0.0294$ for $t_{0}=1 / 46+n / 23$ and (b2) $R_{\mathrm{wp}}=0.0251$ and $R_{\mathrm{p}}=0.0364$ for $t_{0}=0+n / 23$. The superspace group for $\mathrm{Sb}_{87} \mathrm{Te}_{13}$ was assumed to be $R \overline{3} m(00 \gamma) 00$ for both analyses; on the other hand, $F d \overline{3} m$ was applied for $\mathrm{Sb}_{2} \mathrm{O}_{3}$. The diffraction data used for the analysis: $3.0 \leq 2 \theta \leq 30.5^{\circ}$. The standard deviations are shown in parentheses. $B_{n}^{s}$ represents the positional modulation amplitude; $U$ s represent the atomic displacement parameters. Only the first satellites were considered for these analyses.

(a) Incommensurate case $\mathrm{Sb}_{87} \mathrm{Te}_{13}$ :

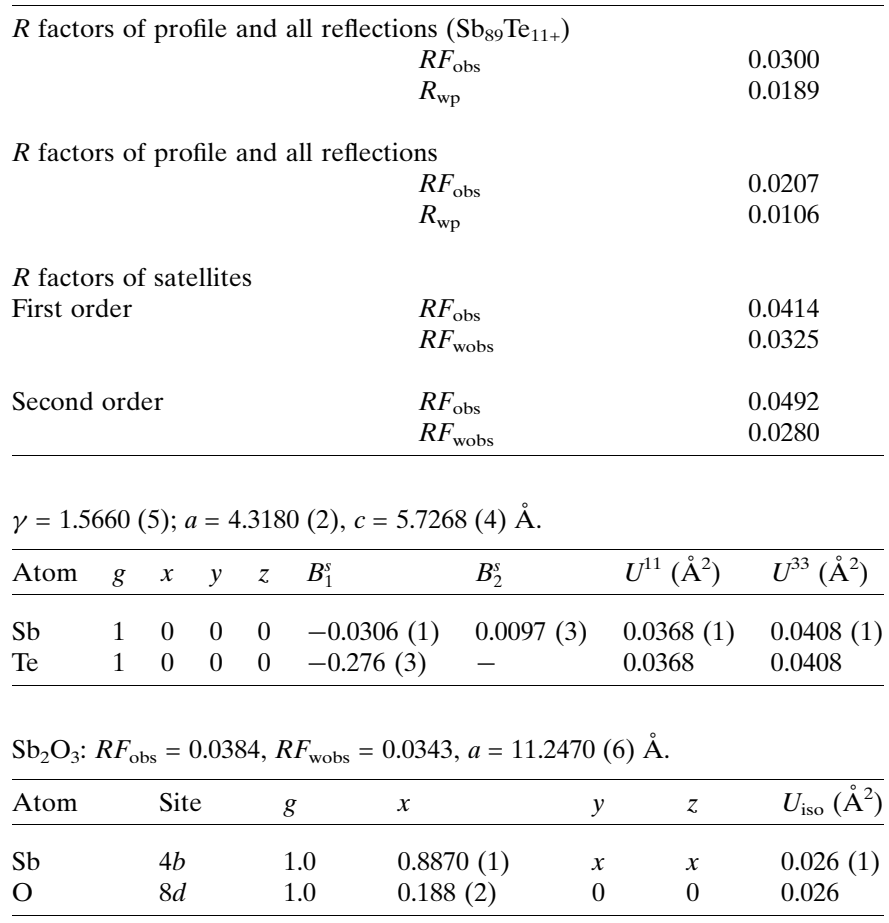

(b1) Commensurate case $\left(t_{0}=\frac{1}{46}+\frac{n}{23} ; n=0,1,2, \ldots\right) . \mathrm{Sb}_{87} \mathrm{Te}_{13}$ :

$R$ factors of profile and all reflections $\left(\mathrm{Sb}_{89} \mathrm{Te}_{11+}\right)$

$$
\begin{array}{ll}
R F_{\text {obs }} & 0.0293 \\
R_{\text {wp }} & 0.0176
\end{array}
$$

$R$ factors of profile and all reflections

$\begin{array}{lll}R \text { Main } & R F_{\text {obs }} & 0.0192 \\ R_{\text {wp }} & 0.0100\end{array}$

$R$ factors of satellites

$\begin{array}{lll}\text { First order } & R F_{\text {obs }} & 0.0424\end{array}$

$\begin{array}{lll} & R F_{\text {wobs }} & 0.0320 \\ \text { Second order } & R F_{\text {obs }} & \\ & R F_{\text {wobs }} & 0.0472 \\ & & 0.0246\end{array}$

$\gamma=36 / 23 ; a=4.3181$ (2), $c=5.7267$ (4) ̊.

\begin{tabular}{lllllllll}
\hline Atom & $g$ & $x$ & $y$ & $z$ & $B_{1}^{s}$ & $B_{2}^{s}$ & $U^{11}\left(\AA^{2}\right)$ & $U^{33}\left(\AA^{2}\right)$ \\
\hline $\mathrm{Sb}$ & 1.0 & 0 & 0 & 0 & $-0.0306(1)$ & $0.0105(3)$ & $0.0368(1)$ & $0.0413(3)$ \\
$\mathrm{Te}$ & 1.0 & 0 & 0 & 0 & $-0.285(3)$ & - & 0.0368 & 0.0413 \\
\hline
\end{tabular}

$\mathrm{Sb}_{2} \mathrm{O}_{3}: R F_{\text {obs }}=0.0360, R F_{\text {wobs }}=0.0314, a=11.2471(6) \AA$.
Table 5 (continued)

\begin{tabular}{lllllll}
\hline Atom & Site & $g$ & $x$ & $y$ & $z$ & $U_{\text {iso }}\left(\AA^{2}\right)$ \\
\hline $\mathrm{Sb}$ & $4 b$ & 1.0 & $0.8870(1)$ & $x$ & $x$ & $0.026(1)$ \\
$\mathrm{O}$ & $8 d$ & 1.0 & $0.188(2)$ & 0 & 0 & 0.026 \\
\hline
\end{tabular}

(b2) Commensurate case $\left(t_{0}=0+\frac{n}{23} ; n=0,1,2, \ldots\right) . \mathrm{Sb}_{87} \mathrm{Te}_{13}$ :

\begin{tabular}{cc}
\hline$R$ factors of profile and all reflections $\left(\mathrm{Sb}_{89} \mathrm{Te}_{11+}\right)$ & \\
$R F_{\mathrm{obs}}$ & 0.0455 \\
$R_{\mathrm{wp}}$ & 0.0257
\end{tabular}

$R$ factors of profile and all reflections

$\begin{array}{lll} & R F_{\text {obs }} & 0.0321 \\ R_{\text {wp }} & 0.0155\end{array}$

$R$ factors of satellites

$\begin{array}{lll}\text { First order } & R F_{\text {obs }} & 0.0719\end{array}$

$\begin{array}{lll} & R F_{\text {wobs }} & 0.0636 \\ & & \\ \text { Second order } & R F_{\text {obs }} & 0.0559 \\ & R F_{\text {wobs }} & 0.0246\end{array}$

$\gamma=36 / 23 ; a=4.3181(2), c=5.7275$ (5) $\AA$.

\begin{tabular}{lllllllll}
\hline Atom & $g$ & $x$ & $y$ & $z$ & $B_{1}^{s}$ & $B_{2}^{s}$ & $U^{11}\left(\AA^{2}\right)$ & $U^{33}\left(\AA^{2}\right)$ \\
\hline $\mathrm{Sb}$ & 1.0 & 0 & 0 & 0 & $-0.0243(2)$ & $0.0065(3)$ & $0.0382(2)$ & $0.0331(4)$ \\
$\mathrm{Te}$ & 1.0 & 0 & 0 & 0 & $-0.326(2)$ & - & 0.0382 & 0.0331 \\
\hline
\end{tabular}

$\mathrm{Sb}_{2} \mathrm{O}_{3}: R F_{\text {obs }}=0.0552, R F_{\text {wobs }}=0.0358, a=11.2478(8) \AA$.

\begin{tabular}{lllllll}
\hline Atom & Site & $g$ & $x$ & $y$ & $z$ & $U_{\text {iso }}\left(\AA^{2}\right)$ \\
\hline $\mathrm{Sb}$ & $4 b$ & 1.0 & $0.8871(2)$ & $x$ & $x$ & $0.021(2)$ \\
$\mathrm{O}$ & $8 d$ & 1.0 & $0.187(3)$ & 0 & 0 & 0.021 \\
\hline
\end{tabular}

four-dimensional structural analyses for the two cases where this Sb-Te crystal took an incommensurately or commensurately modulated structure. As shown in Table 5 these analyses gave almost the same good results, when $\gamma=36 / 23$ ( $n=9, m=$ 1; $\mathrm{Sb}_{87.0} \mathrm{Te}_{13.0}$ ) was applied in the commensurate case (this three-dimensional structure model is shown in Fig. $7 b$ ). However, the results of the commensurate case could be considered somewhat better than those of the incommensurate case ( $c f$. Table $5 a$ with Table $5 b 1$ ), in contrast to the examination of $\mathrm{Sb}_{89} \mathrm{Te}_{11}$. In addition, we can find a clear $t_{0}$ dependence of the $R$ values in the results of the Rietveld analyses performed in the commensurate case ( $c f$. Table $5 b 1$ with Table 5b2). This strongly suggests that this $\mathrm{Sb}_{87} \mathrm{Te}_{13}$ compound eventually obtained a (probably stable) commensurate structure through rearrangement of the atoms from the A7-type atomic configuration after sufficient heat treatment for this material, as observed in the case of $\mathrm{Sb}_{8} \mathrm{Te}_{3}$ (Kifune et al., 2011). Generally, the determination between the commensurate and incommensurate case seems to be beyond the information contained in our powder data. However, we believe that it is very likely that many of these compounds ultimately obtain commensurate structures after sufficient heat treatment. We intend to conduct further experiments and analyses for these materials to reveal their structural features more precisely. 


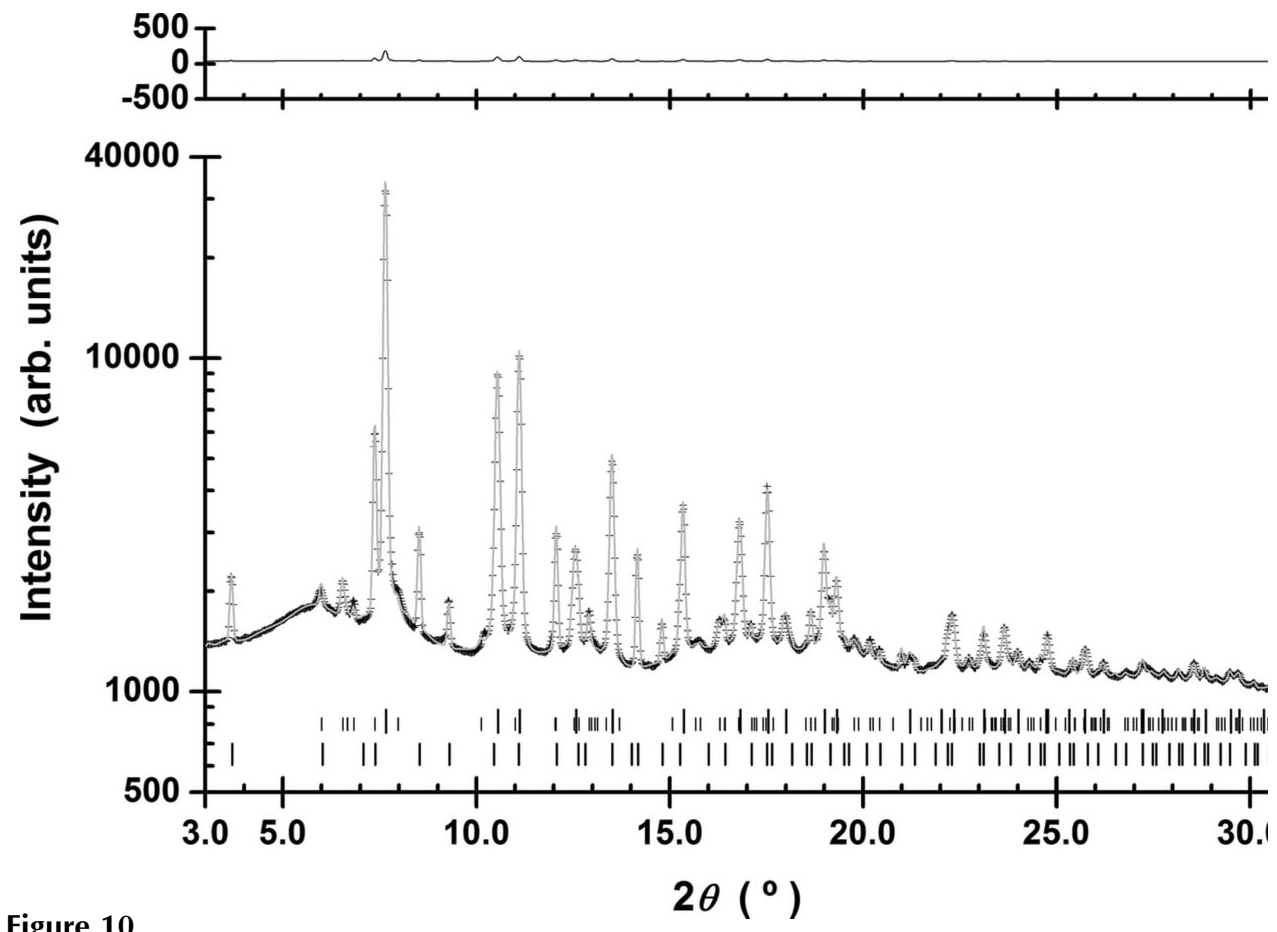

Figure 10

Observed (+) and calculated (gray line) X-ray diffraction profiles for $A_{3} g_{3.4} \operatorname{In}_{3.7} \mathrm{Sb}_{76.4} \mathrm{Te}_{16.5}\left(\mathrm{Sb}_{87} \mathrm{Te}_{13}+\right.$ $\mathrm{Sb}_{2} \mathrm{O}_{3}$ ) at $873 \mathrm{~K}$ by Rietveld analysis in the commensurate case (see Table $5 b 1$ ). This diffraction data were obtained in 1999 at SPring-8 (Matsunaga et al., 2001). A difference curve (observed - calculated) appears at the top of the figure; reflection markers are indicated by vertical spikes below the diffraction patterns. Of the first line of spikes the longer ones are for the peaks of the main reflections of $\mathrm{Sb}_{87} \mathrm{Te}_{13}$, and the shorter ones are for the satellites. Those at the bottom show the peak positions for $\mathrm{Sb}_{2} \mathrm{O}_{3}$.

\section{Conclusions}

An $\mathrm{Ag}_{3.4} \mathrm{In}_{3.7} \mathrm{Sb}_{76.4} \mathrm{Te}_{16.5}$ quaternary amorphous film was first crystallized into an A7-type structure, in which the four types of atoms randomly occupied the atomic sites, right after the phase transformation. However, as the temperature was raised, this single crystalline phase separated into two crystalline phases, $\mathrm{AgInTe}_{2}$ and an Sb-Te binary compound. Of these two crystals, $\mathrm{AgInTe}_{2}$ was stable up to the melting point. In contrast, the latter crystal, $\mathrm{Sb}_{89} \mathrm{Te}_{11}$, had a modulated layer structure, and its modulation vector grew with an increasing in temperature.

The synchrotron radiation experiments were performed on BL02B2 at SPring-8 with the approval of the Japan Synchrotron Radiation Research Institute (proposal Nos. 2010B0084, 2010B1827 and 2011B0030. We express our sincere gratitude to Dr J. Kim at JASRI and to graduate students K. Shakudo, Y. Sato and T. Tachizawa of the Graduate School of Science at Osaka Prefecture University for their assistance with the experiments. The structural models in Fig. 7 were displayed using the Java Structure Viewer (JSV 1.08 lite) created by Dr Steffen Weber.

\section{References}

Clark, G. L. (1955). Applied X-rays. New York: McGraw-Hill. Dušek, M., Petříček, V. \& Palatinus, L. (2010). J. Phys. 226, 012014.
Hoffmann, R. (1988). Solids and Surfaces. New York: VCH Publishers.

Iwasaki, H., Ide, Y., Harigaya, M., Kageyama, Y. \& Fujimura, I. (1992). Jpn. J. Appl. Phys. 31, 461-465.

Karpinsky, O. G., Shelimova, L. E., Kretova, M. A. \& Fleurial, J.-P. (1998). J. Alloys Compd. 268, 112117.

Kifune, K., Fujita, T., Kubota, Y., Yamada, N. \& Matsunaga, T. (2011). Acta Cryst. B67, 381-385.

Kifune, K., Kubota, Y., Matsunaga, T. \& Yamada, N. (2005). Acta Cryst. B61, 492-497.

Kuznetsova, L. A., Kuznetsov, V. L. \& Rowe, D. M. (2000). J. Phys. Chem. Solids, 61, 1269-1274.

Lind, H. \& Lidin, S. (2003). Solid State Sci. 5, 47-57.

Matsunaga, T., Akola, J., Kohara, S., Honma, T., Kobayashi, K., Ikenaga, E., Jones, R. O., Yamada, N., Takata, M. \& Kojima, R. (2011). Nat. Mater. 10, 129-134.

Matsunaga, T., Kojima, R., Yamada, N., Fujita, T., Kifune, K., Kubota, Y. \& Takata, M. (2010). Acta Cryst. B66, 407-411.

Matsunaga, T., Kojima, R., Yamada, N., Kifune, K., Kubota, Y., Tabata, Y. \& Takata, M. (2006). Inorg. Chem. 45, 2235-2241.

Matsunaga, T., Kojima, R., Yamada, N., Kifune, K., Kubota, Y. \& Takata, M. (2007a). Appl. Phys. Lett. 90, 161919-1-3.

Matsunaga, T., Kojima, R., Yamada, N., Kifune, K., Kubota, Y. \& Takata, M. (2007b). Acta Cryst. B63, 346-352.

Matsunaga, T., Kojima, R., Yamada, N., Kifune, K., Kubota, Y. \& Takata, M. (2008). Chem. Mater. 20, 5750-5755.

Matsunaga, T., Morita, H., Kojima, R., Yamada, N., Kifune, K., Kubota, Y., Tabata, Y., Kim, J.-J., Kobata, M., Ikenaga, E. \& Kobayashi, K. (2008). J. Appl. Phys. 103, 093511-1-9.

Matsunaga, T., Umetani, Y. \& Yamada, N. (2001). Phys. Rev. B, 64, 184116-1-7.

Matsunaga, T. \& Yamada, N. (2004a). Phys. Rev. B, 69, 104111-1-8. Matsunaga, T. \& Yamada, N. (2004b). Jpn. J. Appl. Phys. 43, 47044712.

Matsunaga, T., Yamada, N., Kojima, R., Shamoto, S., Sato, M., Tanida, H., Uruga, T., Kohara, S., Takata, M., Zalden, P., Bruns, G., Sergueev, I., Wille, H. C., Hermann, R. P. \& Wuttig, M. (2011). Adv. Funct. Mater. 21, 2232-2239.

Matsunaga, T., Yamada, N. \& Kubota, Y. (2004). Acta Cryst. B60, 685-691.

Nishibori, E., Takata, M., Kato, K., Sakata, M., Kubota, Y., Aoyagi, S., Kuroiwa, Y., Yamakata, M. \& Ikeda, N. (2001). Nucl. Instrum. Methods A, 467-468, 1045-1048.

Petrríček, V. \& Dušek, M. (2000). JANA2000. Institute of Physics, Praha, Czech Republic.

Petříček, V., Dušek, M. \& Palatinus, L. (2006). JANA2006. Institute of Physics, Praha, Czech Republic.

Poudeu, P. F. P. \& Kanatzidis, M. G. (2005). Chem. Commun. pp. 2672-2674.

Rietveld, H. M. (1969). J. Appl. Cryst. 2, 65-71.

Shelimova, L. E., Karpinskii, O. G., Konstantinov, P. P., Avilov, E. S., Kretova, M. A. \& Zemskov, V. S. (2004). Inorg. Mater. 40, 530540. 


\section{research papers}

Shelimova, L. E., Karpinskii, O. G., Konstantinov, P. P., Kretova, M. A., Avilov, E. S. \& Zemskov, V. S. (2001). Inorg. Mater. 37, 342348.

Shelimova, L. E., Karpinskii, O. G., Zemskov, V. S. \& Konstantinov, P. P. (2000). Inorg. Mater. 36, 235-242.

Shelimova, L. E., Konstantinov, P. P., Karpinsky, O. G., Avilov, E. S., Kretova, M. A. \& Zemskov, V. S. (2001). J. Alloys Compd. 329, 5062.
Wolff, P. M. de (1974). Acta Cryst. A30, 777-785.

Wolff, P. M. de, Janssen, T. \& Janner, A. (1981). Acta Cryst. A37, 625636.

Wuttig, M. \& Yamada, N. (2007). Nat. Mater. 6, 824-832.

Wyckoff, R. W. G. (1986). Crystal Structures, Vol. 2. Florida: Robert E. Krieger Publishing Company.

Yamada, N., Ohno, E., Nishiuchi, K., Akahira, N. \& Takao, M. (1991). J. Appl. Phys. 69, 2849-2856. 